\title{
SIMULATIONS OF THE ONSET AND THE EVOLUTION OF CORONAL MASS EJECTIONS
}

\author{
A. De Groof and S. Poedts \\ Centrum voor Plasma Astrofysica \\ Celestijnenlaan 200B, 3001 Leuven, Belgium
}

\begin{abstract}
Simulations of Coronal Mass Ejections (CMEs) evolving in the interplanetary (IP) space from the Sun up to $1 \mathrm{AU}$ are performed in the framework of ideal magnetohydrodynamics (MHD). The aim is to quantify the effect of the background solar wind and of the CME initiation parameters on the evolution and on the geoeffectiveness of CMEs. The shocks and magnetic clouds related to fast CMEs in the solar corona and interplanetary space play a crucial role in the study of space weather. Better predictions of space weather events require a deeper insight in the physics behind them. Different solar wind models are considered in combination with different CME initiation models: magnetic foot point shearing and magnetic flux emergence. The simulations show that the initial magnetic polarity substantially affects the IP evolution of the CMEs influencing the propagation velocity, the shape, the trajectory (and, thus, the geo-effectiveness).
\end{abstract}

\section{INTRODUCTION}

As the sun is a star, composed of electrically charged particles (plasma), its magnetic field makes it a very dynamic, magnetically dominated and highly structured object. The sun shows up as a star with many faces, emitting in a whole series of wavelengths, ranging from low frequency radio waves to high frequency gamma rays. Each wavelength corresponds to a certain temperature layer in the solar atmosphere. Since the solar spectrum is dominated by yellow optical light, emitted by the solar surface and overpowering the light from the outer atmospheric layers, our common view of the sun from Earth consists of a yellowish, bright, but fairly static ball. Moreover the earth's atmosphere filters out almost all but the optical light leaving us with a rather dull view from our nearest star. In order to observe the sun in all its glory one should go out into space. Starting from 1973 with the X-ray telescope on board Skylab and especially with successful satellites like Yohkoh and the Solar and Heliospheric Observatory (SOHO), the sun was found to be highly

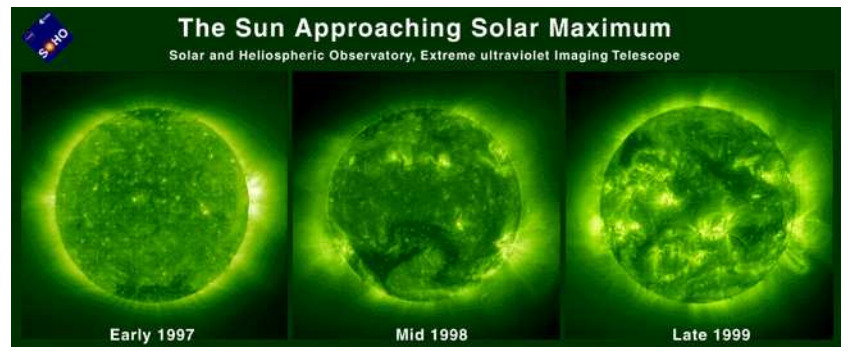

Figure 1: This collection of 3 EIT images (19.5nm), showing the solar corona at 1 million $\mathrm{K}$, illustrates how the level of solar activity has increased significantly towards the solar maximum, attained in 2000.

dynamic and structured by the magnetic field. SOHO, launched in 1995 and still producing an extremely interesting amount of data, is located in the L1 point (on the Sun-Earth line where the gravitational forces from both celestial bodies compensate each other) so that it can observe the sun 24 hours a day. Thanks to the SOHO instruments continuously observing the solar atmosphere in a wide range of wavelengths, the main characteristics and dynamics of the different layers in the solar atmosphere became known.

The first layer, the photosphere, is the visible surface of the sun that we are most familiar with. It is a dense, thin (only $100 \mathrm{~km}$ ), and very bright region mainly emitting in visible light. One of its main characteristics is the presence of dark (because cooler) sunspots at regions with a strong concentration of magnetic field. The number and size of the sunspots follow a cycle of about eleven years, going from solar minimum when the magnetic field is close to a dipole and no sunspots are seen, to solar maximum when the magnetic field is tangled and structured in a complex way and active regions appear showing up as sunspots in the photosphere.

Above the photosphere is the chromosphere, a region of about 2500 kilometers thick. Just prior to and just after the peak of a total solar eclipse, the chromosphere appears as a thin reddish ring. The conspicuous color of the chromosphere (compared to the mostly white corona) led to its name (meaning "color sphere"). The 

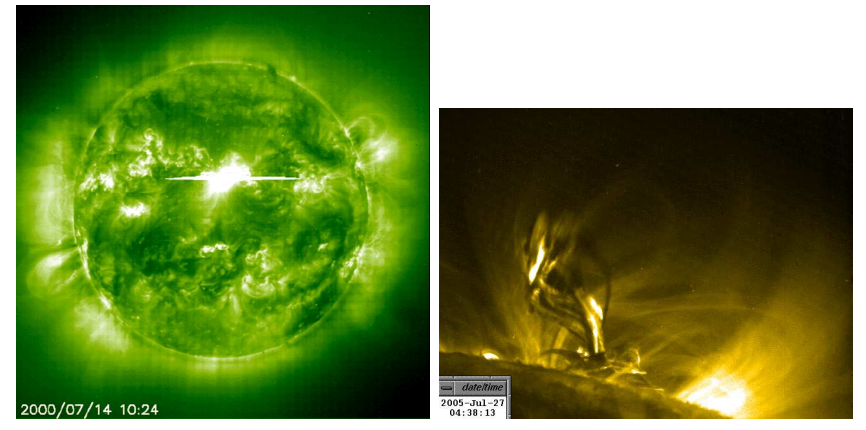

Figure 2: Left: SOHO/EIT 19.5nm image of an X-class solar flare. - Right: TRACE $17.1 \mathrm{~nm}$ observation of a prominence eruption on 27 Jul 2005 (associated with a flare and a CME).

chromosphere is most easily viewed in emission lines such as Hydrogen alpha, where bright regions known as plages, and dark features called filaments are visible. Filaments are the name given to prominences when they are seen on the solar disk.

Finally, the corona is the sun's outer atmosphere. It is visible during total solar eclipses as a pearly white crown surrounding the sun. The corona displays a variety of features including loops (where field lines are connecting back to the surface), helmet streamers (on top of closed field regions), and plumes (in the polar regions). These features are totally dependent on the magnetic field and so the overall shape of the corona changes with the sunspot cycle. Because of the very high temperatures, as much as a few million degrees, the corona emits high energy radiation and can be observed in extreme-ultraviolet light and X-rays. Fig. 1 shows the many faces of the corona in extremeultraviolet light (around 1 million degrees) through the solar cycle.

Furthermore, the corona is the source of the solar wind. The temperature of the corona is so high that the sun's gravity cannot hold onto it. The solar wind streams off of the sun in all directions at speeds of about $400 \mathrm{~km} / \mathrm{s}$. In the polar regions, where the magnetic field lines are not closed in loops but opened up and reaching into space, solar wind speeds up to $700 \mathrm{~km} / \mathrm{s}$ are reached.

\section{THE INFLUENCE OF THE SUN ON EARTH: SPACE WEATHER}

The sun is not a quiet place, but one that exhibits sudden releases of energy. Most of these dynamics occur in the active regions where the magnetic field is the strongest and plasma is shaped in loop like structures. Some coronal loops last for days or weeks but
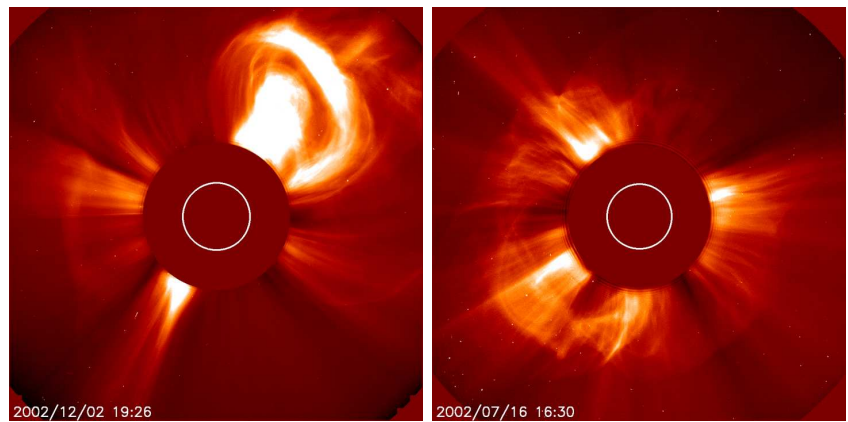

Figure 3: Left: This LASCO C2 image shows a very large CME blasting off into space. It presents the classic shape of a CME: a large bulbous front with a second, more compact, inner core of hot plasma. - Right: In this classic image of an halo CME, taken by LASCO $\mathrm{C} 2$, one can observe particles blasting out from the sun in almost all directions.

most change quite rapidly, often restructuring in other loop configurations. If these magnetic structures become twisted and/or sheared then magnetic field lines cross and reconnect with an explosive release of energy and/or mass as a consequence. One of the most frequently observed events are solar flares: sudden, localized, transient increases in brightness that occur in active regions near sunspots. They are usually most easily seen in $\mathrm{H}$-alpha and X-rays, but may have effects in the entire electromagnetic spectrum (see Fig. 2 left). The X-ray brightness from a large flare often exceeds the X-ray output from the rest of the sun.

Another type of event, the coronal mass ejection (CME), typically disrupts helmet streamers in the solar corona. They can also be associated with prominence eruptions, where the cool, dense prominence material also erupts outward (see Fig. 2 right). As much as $10^{13}$ kilograms of material can be ejected into interplanetary space, on timescales of minutes to hours.

CMEs can be observed by the Large Angle Spectrometric Coronagraph ${ }^{1}$ (LASCO) onboard SOHO. The classic form of a CME is a bright, loop-like leading edge, followed by a less dense cavity and a bright, dense core (Webb et al. (2001), see Fig. 3 left). This type of CME typically lies close to the limb of the sun, and, thus, moves outward at a large angle to the Sun-Earth line. The CMEs heading toward (or away from) the earth, on the other hand, appear as expanding halo-like brightenings surrounding the occulting disk in a coronagraph (see Fig. 3 right). These so-called 'halo CMEs' propagate out in the solar wind, where they may encounter the Earth and influence geomagnetic activity.

\footnotetext{
${ }^{1} \mathrm{~A}$ coronagraph produces an artificial eclipse of the sun by placing an "occulting disk" over the image of the sun.
} 

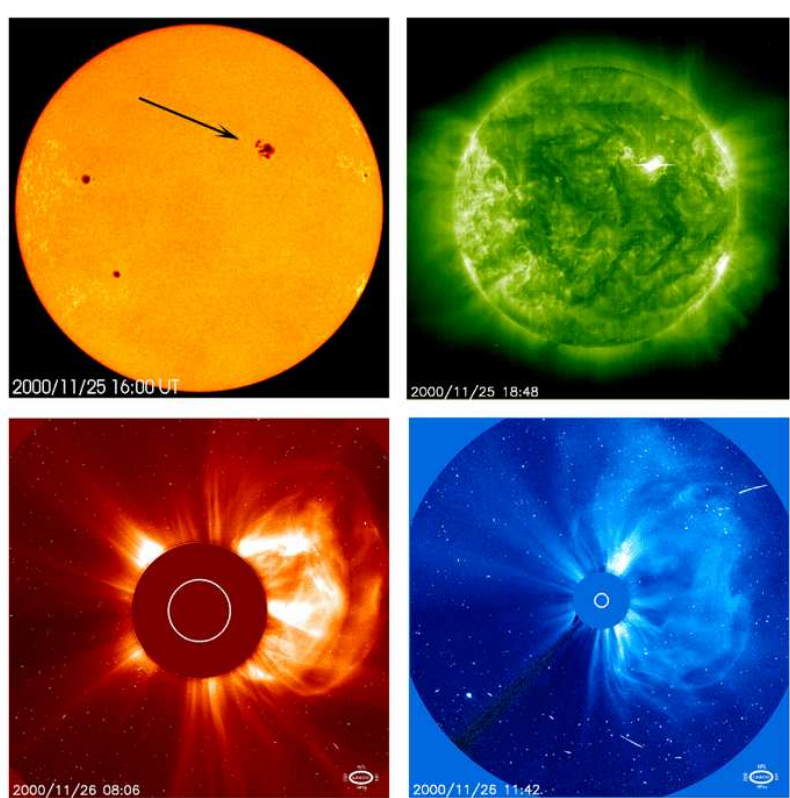

Figure 4: Fireworks in sequence from four $\mathrm{SOHO}$ instruments. This CME, part of a series of 5 in Nov 2000, shows its progress from a sunspot group (MDI), to the flash of a flare (EIT 19.5nm), to a blasting CME seen 14 hours later (LASCO C2), and to a large expanding CME cloud over three hours later.

\section{A. Definition of space weather}

The dynamics of the sun and especially the releases of huge amounts of energy and mass in interplanetary space can influence the earth and her technological systems. This is where the term 'Space Weather' refers to. The official definition of the USA National Space Weather Program (NSWP) Strategic Plan is: "Space Weather refers to conditions on the sun and in the solar wind, magnetosphere, ionosphere, and thermosphere that can influence the performance and reliability of space-borne and ground-based technological systems and can endanger human life or health."

As clear from the definition, the effects of space weather are defined by components on the sun as well as on Earth. The most important solar components are solar flares, halo CMEs and the solar wind. First, as explained before, solar flares are tremendous explosions on the surface of the sun. In a matter of just a few minutes they heat material to many millions of degrees and release as much energy as a billion megatons of TNT. Flares release energy in many forms - electro-magnetic (gamma rays and X-rays), energetic particles (protons and electrons), and mass flows. Flares are characterized and classified by their brightness in X-ray observations. Secondly, halo CMEs which are often associated with a

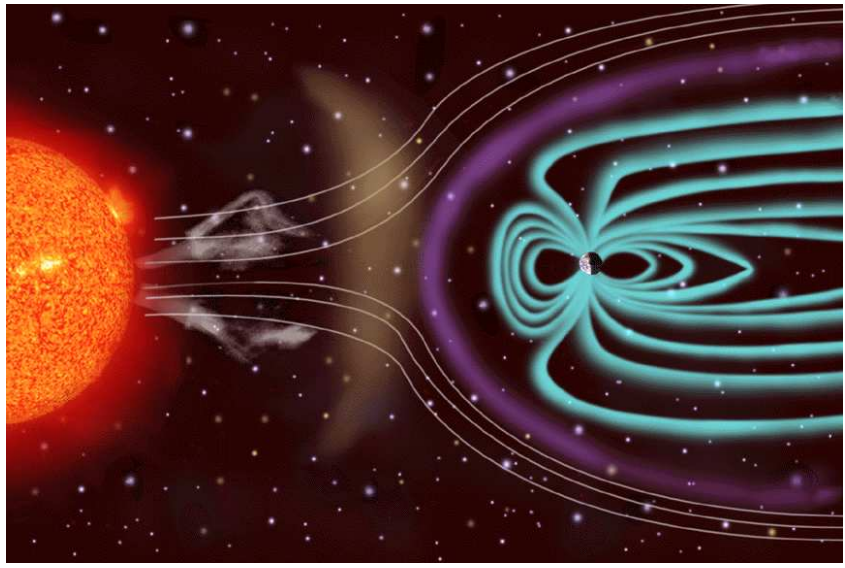

Figure 5: Solar wind shapes the earth's magnetosphere and magnetic storms are illustrated here as approaching Earth. The white lines represent the solar wind; the purple line is the bow shock line; and the blue lines surrounding the Earth represent its protective magnetosphere. The magnetic cloud of plasma can extend to 30 million miles wide by the time it reaches earth.

solar flare (see Fig. 4) can affect the earth by the release of a magnetic cloud of energetic particles. The speeds at which they travel through space range from 400 to more than $2000 \mathrm{~km} / \mathrm{s}$, the average mass of plasma sent to Earth is of the order of $10^{12}-10^{13} \mathrm{~kg}$ and the average energy they represent lies around $10^{24}-10^{25} \mathrm{~J}$ (which is comparable to 5000 atom bombs).

The last crucial solar component of space weather is the solar wind, or better, the locations of the so-called coronal holes where the magnetic field is not closed in loops but reaches into space (open field lines). These regions produce the fastest solar wind (around $700 \mathrm{~km} / \mathrm{s}$ ) and when they are directed towards the earth, this could enhance the effect e.g. CMEs have on the earth's magnetosphere.

The earth on the other hand is not totally unprotected against the endangers of the dynamic sun and the interplanetary space surrounding it. Both the earth's magnetosphere and the ionosphere shield us from any space weather effects. The earth's magnetosphere on one hand produces a magnetic shield that protects earth from the solar energetic particle storms and the solar wind (with or without CMEs) that is continuously flowing around us. The dipole field of the earth is deformed by the solar wind to a typical bullet shape: the magnetosphere is compressed at the sunward side to a distance of 6-10 Earth radii, and dragged out at the night-side (into a magnetotail) to perhaps 1000 Earth radii; no one knows quite how far. This overall shape of the magnetosphere is illustrated in Fig. 5. 
The ionosphere on the other hand protects us from UVradiation and X-rays. Solar radiation at these wavelengths photo-ionizes the neutral atoms in the top layer of the earth's atmosphere and creates in this way an atmospheric layer, the ionosphere, in which the degree of ionization varies greatly with the amount of radiation received from the sun. Apart from the shielding function, the free electrons in the ionosphere are also crucial for radio-communication.

\section{B. Main effects on Earth}

The main effects of space weather (and especially solar weather) on Earth manifest themselves in three groups: radio blackouts, radiation storms and geomagnetic storms.

\section{Radio Blackouts}

Radio blackouts are due to ionospheric disturbances by excessive solar X-ray radiation. The main consequences of the disturbed ionosphere are felt in radio communication. In case of a severe storm, one can experience a high-frequency radio blackout on most or the entire sunlit side of the earth lasting for a number of hours. This can result in lost radio contact with mariners and en route aviators in this sector. Low-frequency radio communication suffers similarly from a solar X-ray storm. Low-frequency navigation signals used by maritime and general aviation systems could experience outages for many hours, causing loss in positioning.

Also satellite navigation might be disrupted by navigation errors in the positioning. Apart from this positioning problems, satellites can also suffer from the fact that the ionosphere gets deformed and often thicker, which leads to drag forces on satellites which fly an orbit just above the (normally sized) ionosphere.

Last but not least, there is a player which always suffers from any form of satellite positioning problems or radio blackouts: the Global Positioning System (GPS).

The strength of a radio blackout is measured by the flux level of X-rays and by the effects on Earth and is classified on a scale ranging from $\mathrm{R} 1$, a minor storm, to R5, an extreme storm.

\section{Solar Radiation Storms}

A solar flare, increasing the amount of energetic particles (flux of ions with energy levels above $10 \mathrm{MeV}$ ), causes an increased level of radiation which could be harmful both for people and technological systems on Earth and in space. First there are biological effects harmful to the human body ${ }^{2}$. Astronauts are strongly

\footnotetext{
${ }^{2}$ Radiation on living cells leads to three possible effects: (1) broken cells can repair themselves, (2) exposed cells can die like many million cells in our body do every day, and (3) cells repair
}

recommended not the perform any extra-vehicular activity (EVA) due to the unavoidable high radiation hazard. Even inside the cabin, the amount of radiation is strongly enhanced. In addition, passengers and crew on commercial jets at high latitudes are exposed to high radiation levels, up to 100 chest X-rays.

On the technological side, satellites may be rendered useless, memory impacts can cause loss of control or may cause noise in image data. Star-trackers may be unable to locate sources and solar panels might get damaged permanently.

Just like radio blackouts, solar radiation storms are classified on a scale from S1 to S5 according to the flux level of particles with energies above $10 \mathrm{MeV}$.

\section{Geomagnetic Storms}

The Earth's magnetic field shielding us from extraterrestrial magnetic clouds can get highly deformed when strong magnetic storms hit the Earth. Depending on the direction and strength of the incoming magnetic field, the geomagnetic field lines can get reconnected and hence opened up for the incoming magnetic flux. This extra flux can cause severe effects on Earth, especially since it induces (additional) electric currents on all devices which are long and conducting. The first victim of these storms are power systems: widespread voltage control problems and protective system problems can occur. In case of an extreme storm (G5) some grid systems may experience complete collapse or blackouts and transformers may get damaged or destroyed. Currents are not only induced in power networks, but also in pipelines, where they can reach hundreds of amps in case of a G5-storm. In addition, geomagnetic storms disturb both high-frequency as lowfrequency radio propagation so that satellite navigation may be degraded for hours or even days. Apart from navigation, orientation and uplink/downlink problems, spacecrafts and satellites are also attacked themselves, in the form of extensive surface charging.

Fortunately, geomagnetic storms also have a positive effect: although aurora are normally visible only at high latitudes, this beautiful effect can sometimes be seen as low as Florida and North Africa.

The strength of a geomagnetic storm (ranging from G1 to G5) is measured by the level of an index $K_{p}$. At several regional stations spread all over the earth, an index $K$ is measured which is related to the disturbance of the geomagnetic field during a period of 3 hours. All regional $K$-indices are combined to a planetary index $K_{p}$, ranging from 0 to 9 , which then determines the strength of the global geomagnetic storm.

themselves but in a wrong way which leads to a bio-physical change. 


\section{Observations and predictions}

Fortunately, people on Earth now realize how important space weather effects are and nowadays many efforts are taken to observe, predict and react on space weather events. Satellites above the earth's atmosphere continuously observe the sun and the interplanetary space in order to detect a storm heading towards us. At this moment, 11 regional space weather centra, spread over the whole world, analyse these observational data and try to model and predict the effects any space weather event could have on Earth. One of them is the Solar Influences Data analysis Centre (SIDC) of the Royal Observatory of Belgium which collects and analyses all data-input and produces predictions and alerts for Western Europe.

The data which are available and used for predictions are summarized and linked to the website http://sidc.oma.be. The most important data to be checked are taken by the EIT and LASCO instruments onboard SOHO and the satellite ACE (both in L1) and by GOES (Geostationary Operational Environmental Satellite) which is orbiting Earth. By means of EITimages and magnetograms (e.g. from SOHO/MDI), growing sunspot groups and increasing magnetic complexity is tracked down and closely followed since these signs lead to an enhanced chance on solar flares. Secondly, after a flare went off, GOES data can give us a measure of the flare strength, from a peak in X-ray flux diagrams eight minutes after the flare went off. After 30 minutes, a jump in the GOES proton flux tells us whether the solar magnetic field lines disturbed by the flare are indeed connected to Earth. If yes, the height of the jump reveals the strength of the solar radiation storm affecting Earth. When a CME is associated to the solar flare, LASCO images reveal whether it is a halo CME and predictions on the speed can be made. This information, together with the solar wind speed measurements by ACE, are then put as input in a CME model which gives us a rough prediction about the arrival time on Earth. The strength of an eventual geomagnetic storm is highly affected by the direction of the magnetic field in the plasma cloud. In case $B_{z}$ (the north-south component) is negative, the geomagnetic field will be highly affected (since opposite field lines can reconnect and thus open the earth's magnetic field for solar influences). Unfortunately, the $B_{z}$ component can often only be measured when it has already reached the Earth's magnetosphere.

The actions which are taken after a solar weather prediction is made, are mainly in the form of space weather alerts. E-mails and text messages with up-todate space weather information are sent to all people or companies with a special interest in space weather news. Furthermore, all possible effects on Earth af-

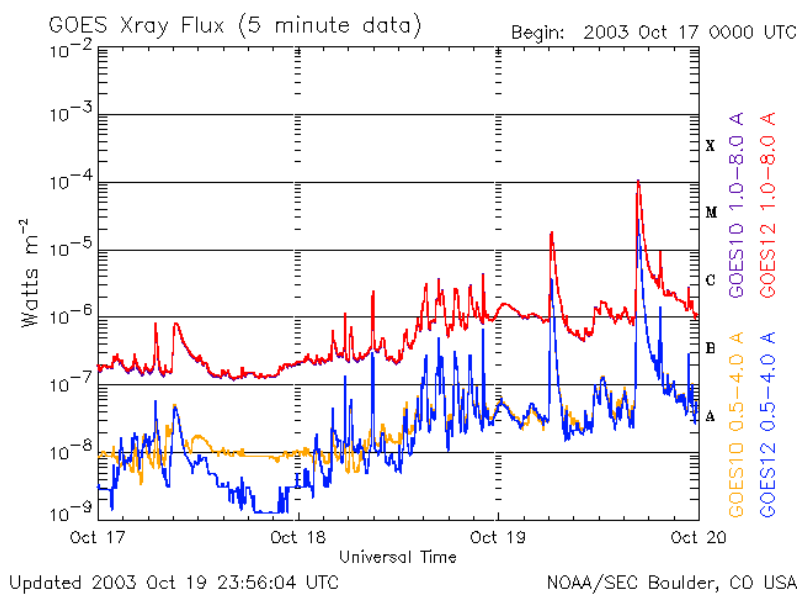

Figure 6: GOES X-ray flux from October 17-20. First the background X-ray emission raised until the C-level. On October 19 two flares went off, one M-flare and one X1.1 flare.

ter the arrival of the solar storm are measured and/or collected from different sources in order to learn more about the possible impact of future storms.

\section{THE 'MONSTER FLARES' OF OCTOBER AND NOVEMBER 2003}

As an example of a very dynamic week on the sun and in the space weather news on Earth, we give the chronological evolution of what happened in autumn 2003 as a result of the explosive behaviour of a few 'monster' sunspot groups on the solar surface (Vanlommel et al. (2004)).

\section{A. Chronological evolution}

It all started on October 19. After one week of total flaring silence, the background X-ray emission of the sun, measured by the satellite GOES, had raised the day before to the top of the $\mathrm{C}_{\text {-level }}{ }^{3}$. In a period like autumn 2003 we would expect a rather low background $\mathrm{X}$-ray emission since the sun was in the declining phase of the 11-year solar cycle. The increase in background emission was related to the appearance of a highly complex and large sunspot group labeled NOAA 484 at the eastern limb of the sun (=left on solar images). The sunspot group area had doubled overnight and space weather observers then know there is an increased risk for a very active period to follow. One day later first an M-flare was observed followed by a rather exceptional

\footnotetext{
${ }^{3}$ The measured $\mathrm{X}$-radiation is classified by intensity in five classes: A, B, C, M, X. Depending on the phase in the solar cycle we are in, the background $\mathrm{X}$-ray emission is situated in the $\mathrm{A}, \mathrm{B}$, or $\mathrm{C}$ level, very exceptionally at level $\mathrm{M}$.
} 

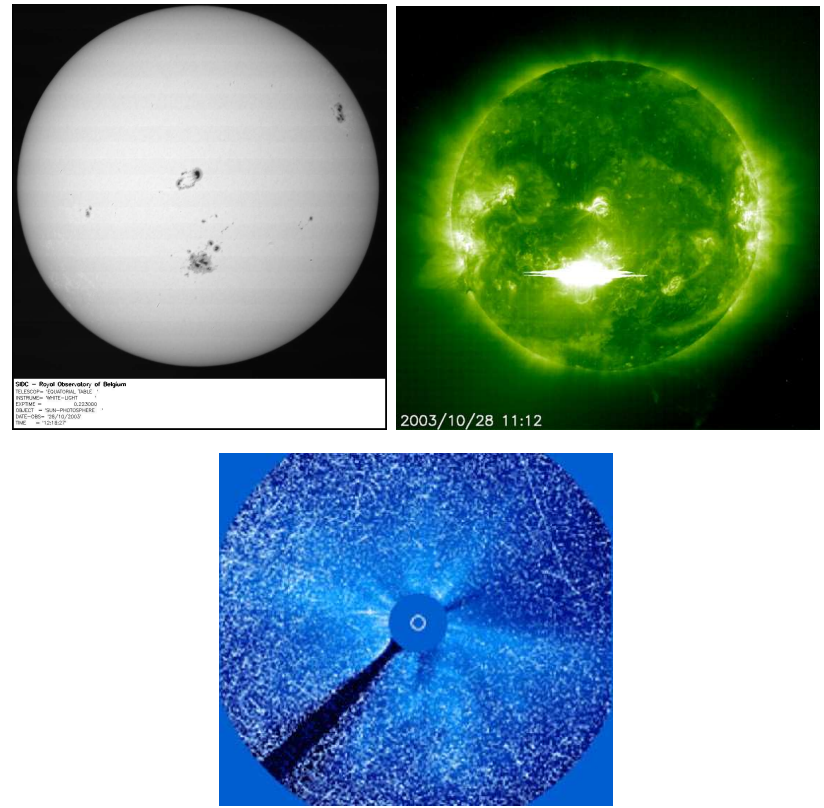

Figure 7: Left: White light image taken from the Royal Observatory of Belgium, showing sunspot group NOAA 486 in the centre of the image, south of the equator.-Right: X17.2 solar flare from the same region, observed by SOHO/EIT in 19.5nm.-Bottom: Noise on the LASCO C3 detector due to the proton storm associated with this flare.

X1.1 flare in the afternoon (see Fig. 6). This was the first in a whole row of explosive events where the Xray emission reached the $\mathrm{X}$-level. The best was still to come.

After the explosive start of region NOAA 484, a new sunspot group NOAA 486 took over in an even more impressing way. On October 23, first a X5.4 solar flare took off, closely followed by a colleague of class X1.1. A few days later, on October 28, the first 'red alerts' were sent around. The monster sunspot group NOAA 486 had just produced an X17.2 solar flare, at that moment the third biggest solar flare ever measured! The solar flare was associated with a severe protonstorm. As the sunspot group was located right in the middle of the solar disk at the time the flare went off (see Fig. 7), the protons were ejected with relativistic speeds in the direction of Earth. Already half an hour later, the particles were detected by satellites in L1, which is located relatively close to Earth. As clear from Fig. 7 (bottom), these protons are observed in EIT and LASCO images as the white noise which troubles the instruments' sight. In the worst case such proton storms can severely damage the satellite (see previous section). In the case of the X17.2 solar flare, the proton flux measured by ACE (Fig. 8) showed a very clear rise. This one was followed

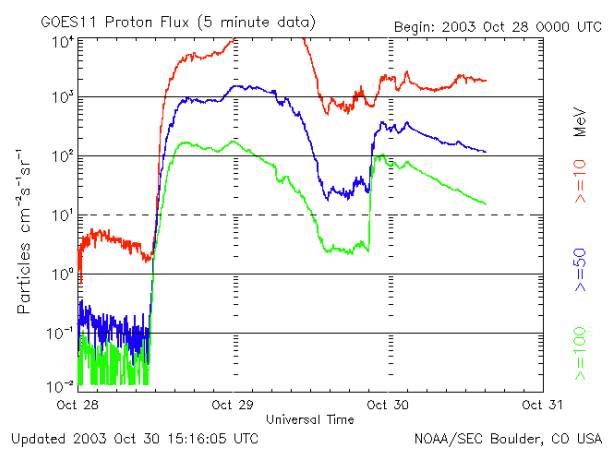

Figure 8: Proton flux measured by GOES, showing two bumps on 28 Oct 2003, due to the X17.2 and X10 flares and on 29 Oct due to an extra X10.1 flare.

by another clear bump due to the following X10 flare on the same day.

Apart from this solar radiation storm, Earth was also attacked by a full halo coronal mass ejection. While a normal CME has a speed around $500 \mathrm{~km} / \mathrm{s}$, this one was ejected towards Earth with an estimated speed of $2152 \mathrm{~km} / \mathrm{s}$. As a result this highly energetic and fast CME would reach Earth extremely fast. In normal conditions, the satellite ACE can warn us when a magnetic cloud will soon collide with the Earth's magnetosphere. The speed of the solar wind measured by ACE, then shows a high jump while the CME is passing by. Unfortunately, ACE could not measure any speeds that day since the instrument was temporarily out of order due to the proton storm passing by earlier. The arrival of the magnetic cloud around 06:00UT on October 29 was only observed by the sudden jump in the $B_{z}$-component of the interplanetary magnetic field which was suddenly strongly negative $(<-60$ nanoTesla). Less than 19 hours after the explosive event on the sun, the CME already reached Earth! As a consequence, a severe geomagnetic storm hit the magnetosphere. A $K_{p}$-index of 9 was measured for the period from 06:00UT until 09:00UT. With such a high value, aurora are quasiguaranteed at heights around Belgium and even more south, but since the CME arrived during the European morning, no special auroral activity was seen. We did not have to wait long for the next opportunity though, since on October 29, the same sunspot group produced another X10.1 flare with associated CME which arrived on October 30 around 16:00UT. The $K$-index was pushed to the top again and that night, polar light was seen in Belgium and around (see Fig. 9).

The sunspots moved more to the west side of the sun (right on solar images) and at November 4, when the group had just passed the western limb, GOES observed another extremely strong solar flare. It was first classified as an X17.4 flare but since the active region was 


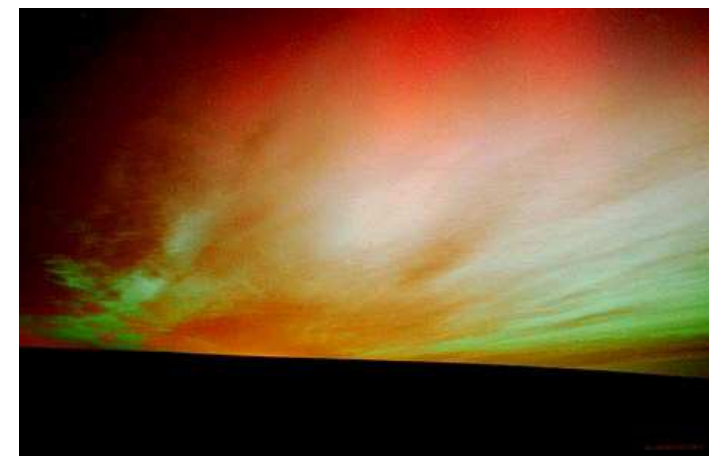

Figure 9: Aurora in Waremme, Belgium on 30 Oct. 2003 (J.M. Maillard).

already behind the limb when the flare took off, it was estimated later as an X28 flare, the biggest ever measured!

\section{B. Main effects on Earth}

The series of solar storms in October/November 2003 led to several effects on Earth. The main ones can be summarized as an $\mathrm{R} 4$ radio blackout, an $\mathrm{S} 4$ solar radiation storm and a G5 geomagnetic storm. Radio communication was highly disturbed due to a high-frequency blackout in several regions, e.g. around Antartica, and the outage of low-frequency navigation signals all around the world led to severe positioning problems of satellites, airplanes, etc. Due to the radiation storms, passengers on pole flights got up to 10 chest x-ray exposures and astronauts were kept inside their vehicles. Satellite operations suffered from memory, orientation and noise problems. Thirdly, the geomagnetic storm caused voltage control problems and electric blackouts, e.g in Sweden and Canada and currents were induced up to hundreds of amperes. Two Japanese satellites got lost and Australia had space craft problems. Radio communication was highly disturbed and even cable TV suffered from the storm. The good news was that auroras where seen at most places in Europe.

\section{SOLAR WIND AND CMES: MODELING AT THE CENTRE FOR PLASMA-ASTROPHYSICS}

In order to improve the geomagnetic storm predictions, studies on CME evolution are really important. At the Centre for Plasma Astrophysics, simulations of Coronal Mass Ejections (CMEs) evolving in the interplanetary (IP) space from the sun up to $1 \mathrm{AU}$ are performed in the framework of ideal magnetohydrodynamics (MHD) by the means of a finite volume, explicit solver. The aim is to quantify the effect of the background solar wind and of the CME initiation parameters, such as the initial magnetic polarity, on the evolution and on the geo-effectiveness of CMEs. In what follows we explain the strategy followed by CPA in order to model CME initiation and evolution in an as objective way as possible. First, three different solar wind models are reconstructed using the same numerical grid and the same numerical scheme. Then, different CME initiation models are considered: magnetic foot point shearing and magnetic flux emergence. For the fast CME evolution studies a very simple CME model is considered: a high density and high pressure magnetized plasma blob is superposed on a background steady state solar wind model with an initial velocity and launch direction.

\section{A. The solar wind models}

We reconstructed three models for the solar wind that are often used in the literature. All three the models are axi-symmetric (2.5D) and were reconstructed with the same numerical code, on the same grid, and with the same boundary conditions such that the only difference between the winds lies in the physics included in the equations. The simulation domain extends to $30 R_{\odot}$ for the $\mathrm{CME}$ initiation studies and to $1 \mathrm{AU}$ for the CME evolution studies.

The first model is a classical polytropic model with polytropic relation: $p=\rho^{\gamma} / \gamma$, where $\gamma=1.05$. This wind model is fairly popular in the literature in spite of its remarkable shortcomings. It has e.g. a too small ratio of polar versus the equatorial wind velocity. Wind model 2 is a reconstruction of the MHD wind model used and described by Manchester et al. (2004a), where the full set of ideal MHD equations is solved. It is obtained by means of an extra heating source term in the energy equation which, in normalized form, is given by:

$$
Q=\rho q_{0} \exp ^{-\frac{(r-1)^{2}}{\sigma^{2}}}\left(T_{0}-\gamma \frac{p}{\rho}\right),
$$

with the profiles of $T_{0}$ and $\sigma$ having both a $\theta$ - and $r$ dependence. This heating source term yields a much better wind model than the polytropic wind, with the right velocity ratio. The third model is again a polytropic model but now including an additional pressure gradient due to Alfvén waves. The extra Alfvén wave pressure, $p_{w}$, in the momentum equation is determined by:

$$
\frac{\partial \epsilon_{ \pm}}{\partial t}+\nabla \cdot\left[\left(\mathbf{v} \pm \mathbf{v}_{\mathbf{A}}\right) \epsilon_{ \pm}\right]=-\frac{\mathbf{1}}{\mathbf{2}} \epsilon_{ \pm} \nabla \cdot \mathbf{v}
$$

where $\epsilon_{ \pm}$is the average Alfvén wave density for the parallel and anti-parallel propagating waves and $\mathbf{v}_{\mathbf{A}}$ is the Alfvén velocity. The three winds are illustrated in Fig. 10 showing the density (green scale), the magnetic 

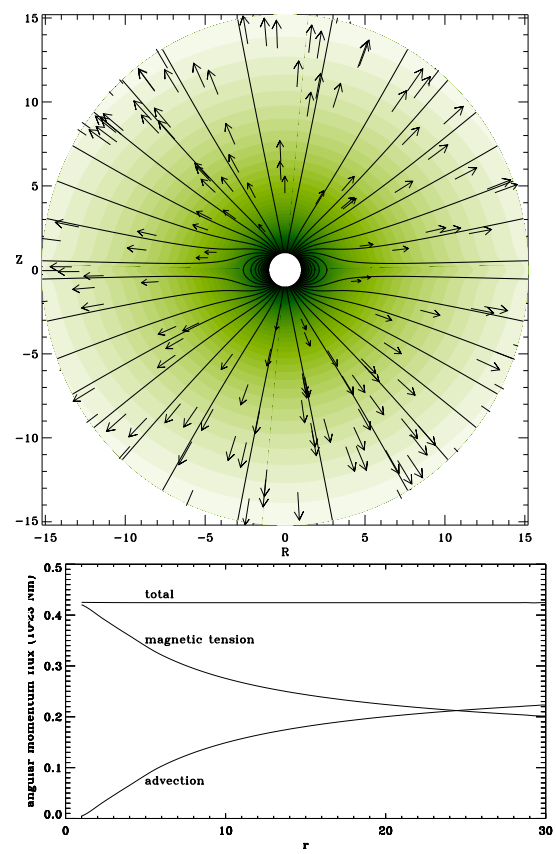
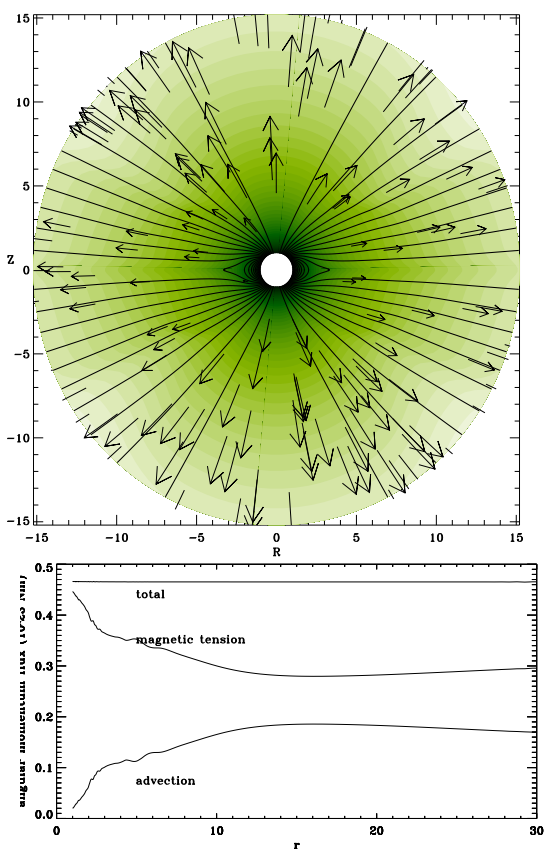
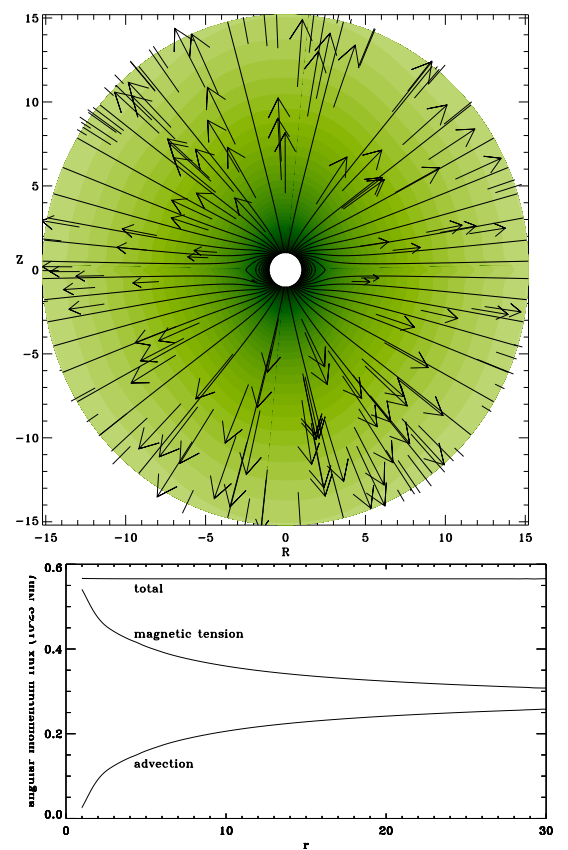

Figure 10: The three steady wind solutions (upper row) and the corresponding total angular momentum fluxes (lower row). Left: the (standard) polytropic model with $\gamma=1.05$, middle: MHD wind model with energy source term, right: polytropic MHD wind model with Alfvén wave heating term. The green scale represents the density (log-scale), the black lines are magnetic field lines, arrows denote the radial velocity.

field lines, the velocity vectors, and the corresponding angular velocity fluxes (AMF). The second wind model was extended to $1 \mathrm{AU}$ for the $\mathrm{CME}$ evolution studies.

\section{B. CME initiation models}

In this paragraph the sub-problem of the CME initiation is handled. Two popular models for CME initiation are foot point shearing and magnetic flux emergence (or cancellation). Both these initiation models are superposed on the different wind models to examine effect of the background wind on the CME initiation. The effect of the initiation parameters (e.g. shear velocity, flux emergence rate, etc.) on the development of the CMEs is also investigated.

\section{B.1 Foot point shearing}

In order to trigger an instability of the magnetic structure $(\mathrm{CME})$ the footpoints of the magnetic field are sheared by adding an extra longitudinal velocity at the solar surface in accordance to Mikic \& Linker (1994). The amount of added longitudinal velocity is given by

$$
v_{\varphi}^{0}=v_{0}(t) \Theta \exp \left[\left(1-\Theta^{4}\right) / 4\right],
$$

where $\Theta=(\theta-90) / \Delta \theta_{m}, \theta$ is the co-latitude in degrees, and $v_{0}(t)$ a function that specifies the time profile. The simulation stops when a time $t_{\max }=180 \mathrm{~h}$ is reached. The shearing reaches its maximum value $\Delta \theta_{m}$ degrees above and below the equator. In the simulations performed we took $\Delta \theta_{m}$ equal to $20^{\circ}$ and the maximum shear velocity varied between values of 3,6 or $9 \mathrm{~km} / \mathrm{s}$. The three different models for the solar wind, described above, were used as background wind.

In Fig. 11 the relative density is shown for successive snapshots in the evolution of the CME formation. The background wind is the standard polytropic model and the shear velocity measures $6 \mathrm{~km} / \mathrm{s}$. By shearing the footpoints, the helmet streamer starts swelling until it becomes unstable and a flux rope is formed. Although the ideal MHD equations are solved, numerical dissipation cannot be excluded and allows the formation of a flux rope by reconnection processes. The relative density is given by $\left(\rho_{t o t}-\rho_{w}\right) / \rho_{w}$, with $\rho_{t o t}$ the total density and $\rho_{w}$ the density of the stationary background wind.

The model for the background wind affects the time of formation of the flux rope. This can be seen in Fig. 12 where a plot is shown of the evolution of the total magnetic energy in time and the maximum in magnetic energy is reached right before the formation of the flux rope. The amount of magnetic energy is expressed with 

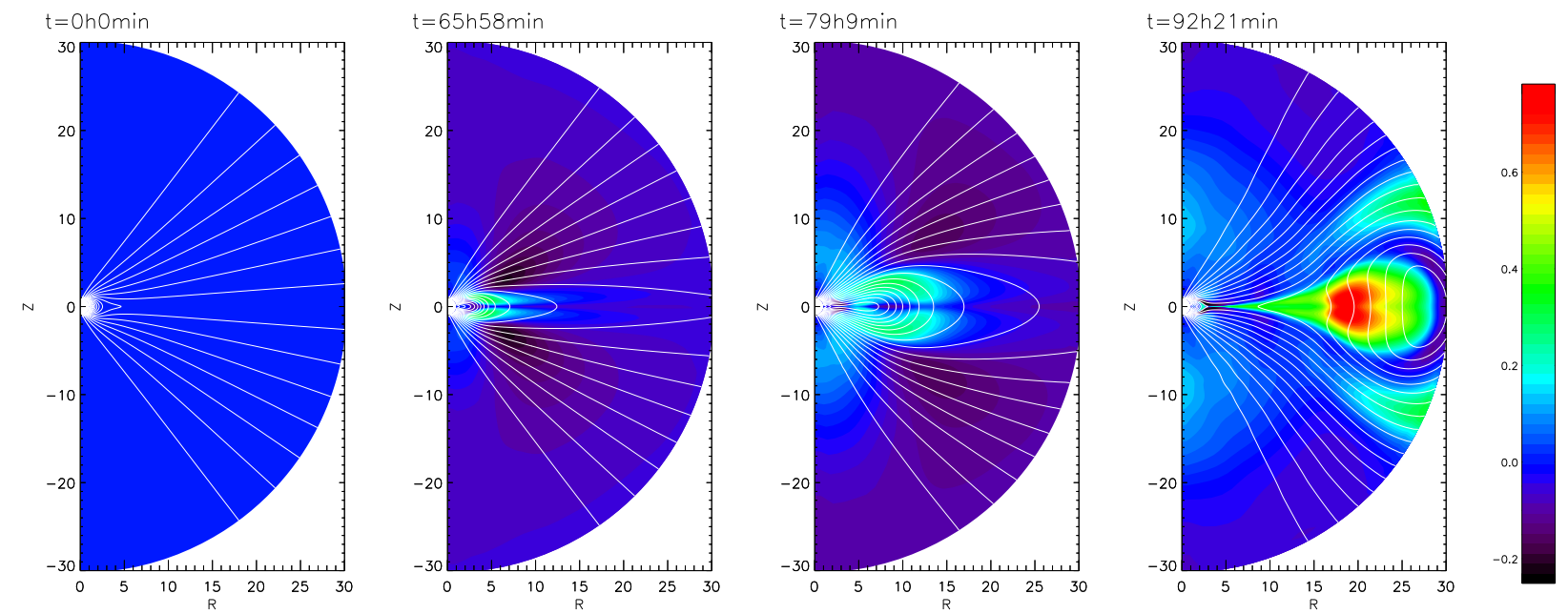

Figure 11: Evolution of the relative density and formation of a flux rope by foot point shearing. Wind model 1, $v_{\phi}^{\max }=6 \mathrm{~km} / \mathrm{s}$

respect to the total amount of magnetic energy in the stationary background wind. Because the shearing is a continuous process, several flux ropes will be formed.

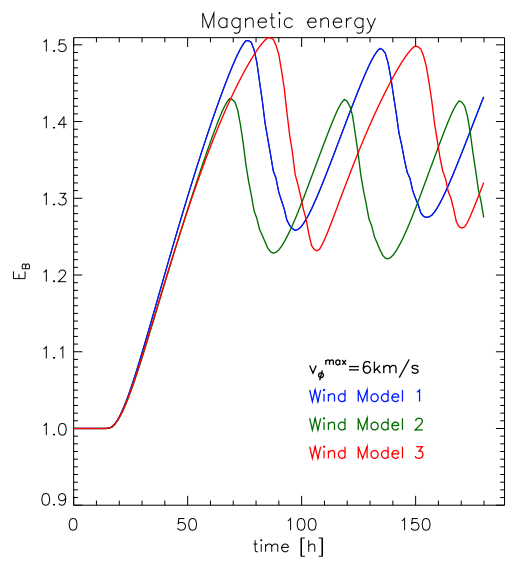

Figure 12: The evolution of the magnetic energy in time, for maximum shear velocity of $6 \mathrm{~km} / \mathrm{s}$.

The faster the shearing, the earlier the flux rope is formed. Also the time-interval between the succeeding flux ropes scales with the shearing velocity. The shear velocity also determines if a flux rope will be formed or not. Shearing at a too low velocity will not lead to the formation of a flux rope. This is the case for shearing at $3 \mathrm{~km} / \mathrm{s}$ in wind model 2 and model 3 . The amount of extra longitudinal velocity added also influences the velocity at which the flux rope moves through interplanetary space. This can be seen in the left part of Fig. 13 where the velocity of the center of the flux rope is plotted against position of the flux rope center. This curve is obtained by taking the derivative with respect to time of the height-time curves of the center of the flux rope. From this we can see that the faster the field lines are sheared, the faster the flux rope is moving. Also the background wind has an influence on the velocity of the flux rope. This can be seen on the right side of Fig. 13. For the given shearing velocities it turns out impossible to create fast CMEs with this initiation mechanism.

\section{B.2 Magnetic flux emergence}

CMEs can also be triggered by the emergence of additional magnetic flux of the same or the opposite polarity as the overlying magnetic field. Dubey et al. (2005) considered a flux rope in a dipole magnetic field with a density that is 200 times higher than at the solar surface. The flux rope is initially kept stable by means of a line current (cf. Chen \& Shibata (2000)). We then allow magnetic flux of the opposite polarity to emerge from the solar surface at a certain rate. This additional magnetic flux breaks the force balance and causes the CME to be launched. The evolution parameters, such as the velocity and the acceleration, depend on the flux emergence rate and on the total amount of flux that is emerged and we obtain velocities in the order of 350 to $400 \mathrm{~km} / \mathrm{s}$, which is precisely in the range of what is observed for the majority of the CMEs. However, Dubey et al. (2005) were not able to create fast CMEs (with velocities $>1000 \mathrm{~km} / \mathrm{s}$ ) in this way. The next steps will, therefore, be to extend the parameter study and to replace the background dipole magnetic field by 
a solar wind model.

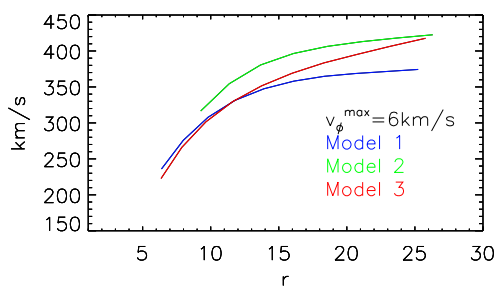

Figure 13: Velocity of the flux rope in the different wind models for $v_{\phi}^{\max }=6 \mathrm{~km} / \mathrm{s}$.

\section{B.3 'Density driven' CMEs}

In order to study the propagation of fast CMEs and CME shocks in the interplanetary space from the solar corona up to $1 \mathrm{AU}$, a very simple model is used to generate the CMEs in the present paper. This model is far to be a state-of-the-art CME initiation model with very complex features, but it gives us an easy way to study the importance of the initial magnetic polarity of the CME. A high density and high pressure magnetized plasma blob is superposed on the background steady state solar wind model. The CMEs are launched at a certain velocity $v_{c m e}$, in a prescribed radial direction $\theta_{c m e}$. The velocity and density profile in the initial disturbance are both of the form:

$$
y=\frac{y_{c m e}}{2}\left(1-\cos \pi \frac{d_{c m e}-d}{d_{c m e}}\right),
$$

where $y$ indicates the density $\rho$ or the radial velocity $v_{r}, y_{c m e}$ is the maximum density or radial velocity in the plasma bubble, $d_{c m e}$ is the radius of the bubble and $d$ the distance to the center of the bubble. The CMEs are further characterized by a given density, magnetic field strength and magnetic polarity. The initial CME magnetic field and the background wind magnetic field can have the same or the opposite polarity. We call it respectively an inverse and a normal CME, according to the nomenclature of Low \& Zhang (2002) (see Chané et al. (2005) for more details about the CME initiation).

For the CMEs discussed in the present paper, the radius of the initial plasma blob, $d_{c m e}$, is $0.29 R_{\odot}$ and its center $d$ is located at $1.5 R_{\odot}$. In the center of the initial perturbation, the velocity is about $1000 \mathrm{~km} / \mathrm{s}$ and the density is 5 times higher than the density on the surface of the Sun. In the initial plasma blob, the maximal magnetic field strength is $\sim 3.44 \mathrm{G}(\sim 0.344 \mathrm{mT})$. In all the numerical simulations discussed in this paper, the CMEs have exactly the same initial density, pressure, velocity and magnetic strength. The only difference will be the initial magnetic polarity: inverse or normal. In the following section, the influence of the initial magnetic polarity on the time of arrival, on the evolution path and on the geo-effectiveness of the ICMEs will be investigated.

\section{CME EVOLUTION}

It turns out that the polarity of the flux rope magnetic field has a great influence on the evolution of the CME. The polarity influences the mass distribution inside the CME, the spread angle, the evolution path, and also the velocity of the shock front. When launching a magnetized CME outside the equatorial plane the magnetic forces push the CME towards or away from the equator, depending if the polarity of the flux rope is inverse or normal. Also it is seen that the normal magnetized CME moves slightly faster than the magnetized inverse. This has consequences for predicting the time of arrival and geo-effectiveness of a CME.

In spite of the rather simple and naive CME model used in these simulations, they yield some very good and realistic results. First of all we were able to reproduce the magnetic topology predicted by Low and Zhang (2002). Behind the CME, in the equatorial plane, reconnection processes occur which lead to a gradually re-building of the helmet streamer and yield back flows along the helmet streamer, very much like seen in observations. Another interesting fact is that we can extend these CME simulations to $1 \mathrm{AU}$ and that we can reproduce some of the cases in the ACE database, where the strength jump in magnetic field, the profile for the velocity, and the time of arrival are matching surprisingly well.

\section{A. Characteristics of the CMEs}

In this subsection, we will try to point out the characteristic shape (density repartition and magnetic field configuration) of the normal and the inverse CMEs. Figure B.3 shows an inverse and a normal CME launched in the equatorial plane, travelling in the IP space. Clearly the shock front travels slower close to the equator resulting in a strange shape transformation of the CMEs. This is due to the wind model we are using which presents a higher density and a lower velocity on the equator. After 9h36min the magnetic cloud still has an almost circular shape. But after 55h11min the magnetic cloud is so compressed that it is hard to recognize the Low and Zhang configuration and the CME evolution is thus far from self-similar. The density repartition of an inverse and a normal CME is, in fact, quite similar at 1 AU. They are both characterized by a shock front evolving in front of the magnetic cloud which contain the main part of the density. The magnetic field 


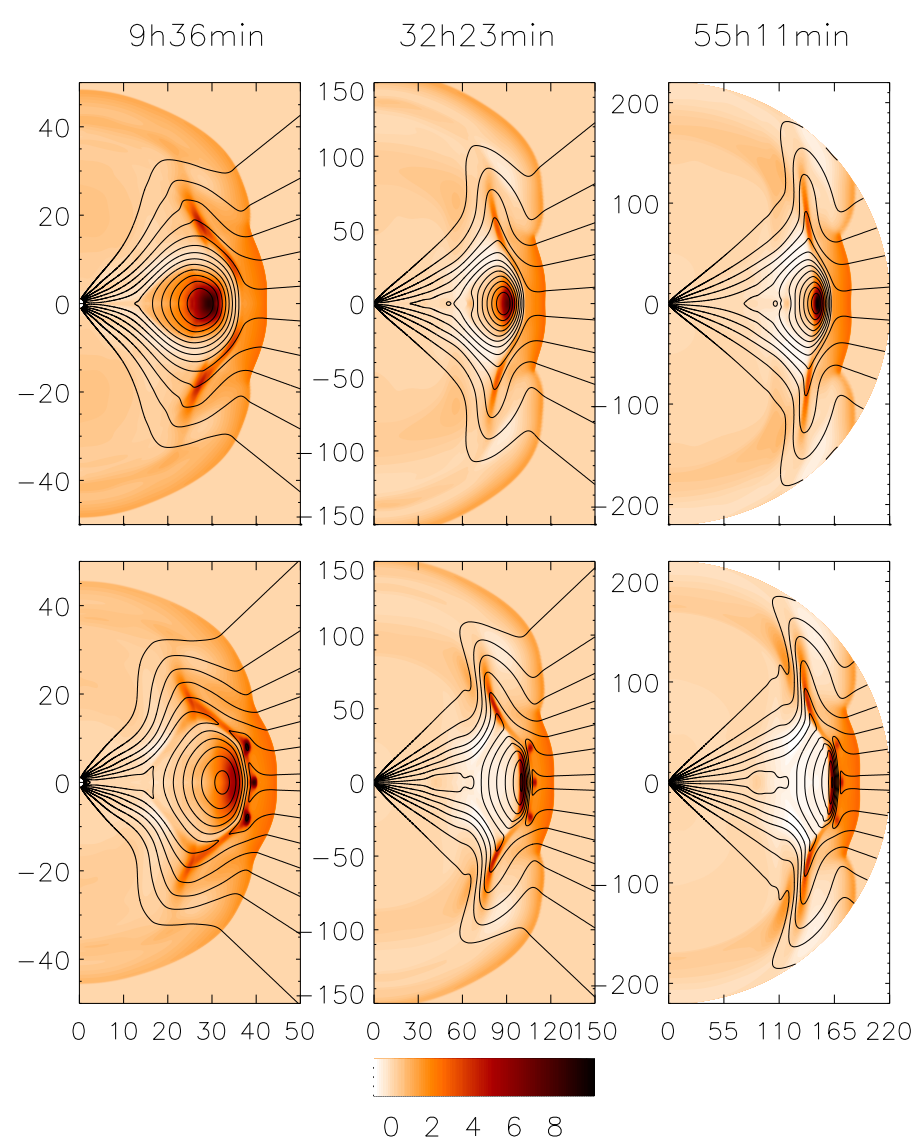

Figure 14: Contour plots of the relative density distribution for a $\mathrm{CME}$ launched in the equatorial plane $\left(\theta_{\text {ini }}=0^{\circ}\right)$. Upper row: inverse initial magnetic polarity, Lower row: normal initial magnetic polarity. Left column: after 9h 36min, Central column: after 32h 23min, Right column: after $55 \mathrm{~h} 11 \mathrm{~min}$. The black lines represent the projected magnetic field lines. The relative density is defined as follows: $\bar{\rho}=\left(\rho-\rho_{\text {wind }}\right) / \rho_{\text {wind }}$.

lines are not that different for the two CMEs although the magnetic cloud seems to be more deformed (less circular) for the normal CME.

Figure 15 mimics the data obtained by a spacecraft at $1 \mathrm{AU}$ when these CMEs would pass it. One can see that the normal CME shock front arrives at 1 AU only four hours before the inverse shock front. The inverse CME displays a strong southward magnetic field which may lead to a magnetic storm and the normal CME displays a strong northward magnetic field which normally does not cause a magnetic storm. The velocity of the normal CME is a little bit higher and so this CME reaches the Earth a few hours earlier than the inverse CME.

\section{B. Evolution path}

When a CME travels in the IP space, it is really important to know its trajectory, i.e. whether its evolution path is a straight line or whether it deviates from this line and, if so, how much. In some cases, this information can be crucial to predict if a CME will hit or miss the Earth. Chané et al. (2005) have studied the evolution path of magnetized CMEs up to $30 R_{\odot}$ and have shown that the initial magnetic polarity of the CME flux rope affects its evolution path substantially: normal CMEs are deviated towards the pole while inverse CMEs are deviated towards the equatorial plane, which means that the probability to hit the Earth should be higher for inverse CMEs. We will show that these conclusions remain partially true up to 1 AU. Figure 16 shows the results of several simulations for inverse and normal CMEs launched at $10^{\circ}, 30^{\circ}$ and $60^{\circ}$. For the CMEs at $30^{\circ}$ and $60^{\circ}$, the normal CMEs are deviated towards the pole while the inverse CMEs are deviated towards the equatorial plane. Surprisingly the inverse 

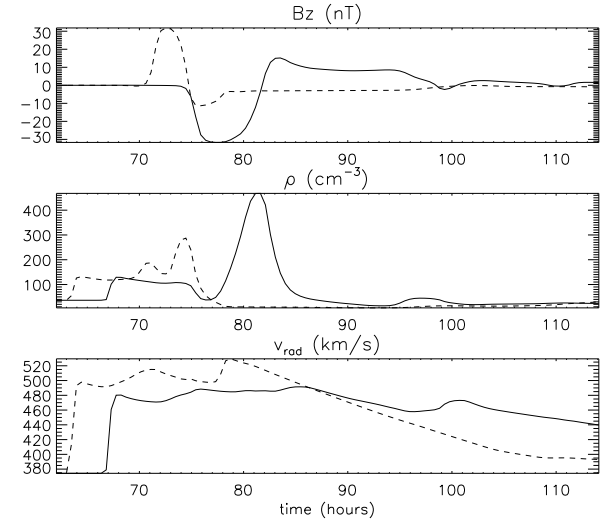

Figure 15: Evolution of the magnetic field, density and velocity in time in the equatorial plane $\left(\theta=0^{\circ}\right)$ at a position of $215 R_{\odot}$ for a CME launched in the equatorial plane $\left(\theta_{\mathrm{cme}}=0^{\circ}\right)$; dashed lines: normal CME; full lines: inverse CME.

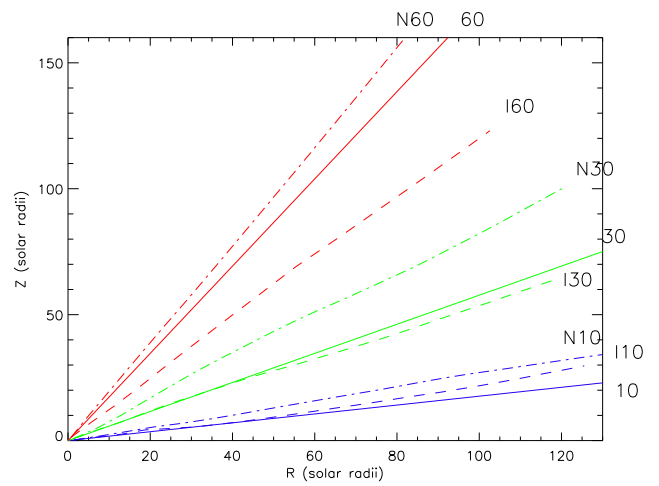

Figure 16: Evolution path of the center of relative mass for inverse (dashed lines) and normal (dasheddot lines) CMEs. The CMEs were launched on $10^{\circ}$, $30^{\circ}$, and $60^{\circ}$. The solid lines show the initial launch angles.

CME launched at $10^{\circ}$ is deviated away from the equatorial plane. This is caused by the density repartition in the wind. The higher density close to the equator acts like a natural shield and protects the Earth from the incoming CMEs. However, the density of this wind model is too high in the equatorial plane (see below) and this probably amplifies this phenomenon. Nevertheless, in our simulations, an inverse CME is always traveling closer to the equatorial plane than a normal one and is thus more dangerous for the Earth.

\section{CONCLUSIONS}

In this paper, we first presented three solar wind models. We discussed CME initiation models based on magnetic foot point shearing and magnetic flux emergence. In spite of extensive parameter studies, however, these models were not able to produce the fast CMEs that create shock waves in the IP space and are, therefore, important for space weather. For the CME evolution studies we thus applied the much simpler, socalled 'density-driven', CME model. We then extended the study of Chané et al. (2005) on the influence of the initial magnetic polarity on the evolution of CMEs into the IP space by extending the computational domain up to $1 \mathrm{AU}$. We have shown that the evolution path of the CMEs was strongly related to the initial magnetic polarity. An example was given where an inverse CME hits the Earth and where the same CME but with a normal magnetic polarity missed the Earth. We have also shown that the time of arrival was influenced by the initial magnetic polarity: a normal CME propagates faster than an inverse $\mathrm{CME}$ and thus reached the Earth a few hours earlier. Last but not least, the inverse CMEs display a strong southward magnetic field (a well known source of magnetic storms) at $1 \mathrm{AU}$. The geo-effectiveness of a CME is thus strongly related to its magnetic polarity.

Acknowledgments These results were obtained in the framework of the projects GOA 2004/01 and OT 02/57 (K.U.Leuven), G.0451.05 (FWOVlaanderen) and 90203 (ESA Prodex 8).

\section{REFERENCES}

Vanlommel P., Van der Linden R., Robbrecht E., et al., 2004, Heelal, 49, 79

Webb D.F., Crooker N., Plunkett S., Cyr O., 2001, In: et al. P. (ed.) Space Weather: Progress and Challenges in Research and Applications, vol. GM125 of AGU, 123

Chané, E., Jacobs, C., van der Holst, B., Poedts, S., \& Kimpe, D. 2005, A\&A, 432, 331

Chen P.F., Shibata K., 2000, Astrophys. J., $\mathbf{5 4 5}, 524$.

Dubey, G., Poedts, S., Van der Holst, B., 2005, Proc. Solar Wind11/Soho 16, Whistler, Canada, 11-17 June, 2005, ESA SP-592, in press (2005).

Low, B. C. \& Zhang, M. 2002, ApJ, 564, L53

Manchester, W. B., Gombosi, T. I., Roussev, I., et al. 2004a, Journal of Geophysical Research (Space Physics), 109, 1102

Mikić Z., \& Linker J.A., 1994, APJ 430‘, 898 